\title{
ANNOTATION APPROACH FOR DOCUMENT WITH RECOMMENDATION
}

\author{
Priyanka Channe and Bhagyashree Dhakulkar
}

${ }^{1}$ Department of Computer Engineering, Savitribai Phule Pune University, Pune, India.

\begin{abstract}
An enormous number of organizations generate and share textual descriptions of their products, facilities, and activities. Such collections of textual data comprise a significant amount of controlled information, which residues buried in the unstructured text. Whereas information extraction systems simplify the extraction of structured associations, they are frequently expensive and incorrect, particularly when working on top of text that does not comprise any examples of the targeted structured data. Projected an alternative methodology that simplifies the structured metadata generation by recognizing documents that are possible to contain information of awareness and this data will be beneficial for querying the database. Moreover, we intend algorithms to extract attribute-value pairs, and similarly devise new mechanisms to map such pairs to manually created schemes. We apply clustering technique to the item content information to complement the user rating information, which improves the correctness of collaborative similarity, and solves the cold start problem.
\end{abstract}

\section{KEYWORDS}

Document Annotation, Adaptive Forms, Collaborative Filtering, Mapping Attribute-Value, Attribute Suggestion.

\section{INTRODUCTION}

When natural disaster events happen, public users are eager to know maximum information about them and curiously they look out for related facts frequently. Such as, what is the severity of the disaster of storms or the magnitude of the earthquake? Searchers are also involved in knowing regarding the damage affected by these natural adversity, e.g., number of people dead or number of homes destroyed. The Observer needs to obtain fresh information about events to show a structured summary of such events.

Now a day there are a large number of institutes that provides applications where users can create and share their data with a textual description of their services, products and actions. Some applications are: user blog, scientific, social network, network management systems, content management systems, etc. are used to share user data and annotate that data using some tag in an informal way. There are various annotation techniques that allow retrieving subsequent information finding. Many annotation schemes permit keyword annotation in which a user may annotate a weather report by means of a tag such as "Storm Category 3", such type of annotation is called as "untyped" keyword annotation.

In social tagging applications the Tag recommenders may contribute users by means of tagging procedure with the suggestion of a set of tags so that users are like to make use of a web resource. The tags provide a meaningful description of the objects, and permit the user to arrange and index content. This becomes even additional vital, once coping with multimedia system objects that give little or no textual context, like bookmarks, photos and videos. 
The purpose is to resolve these annotations and ranking issues. Collaborative Adaptive Data Sharing platform (CADS) using content value and query value and probabilistic Tag Relevance is proposed. A key contribution of this paper is the actual use of the query workload to scrutinize the text of the document, in addition to direct the annotation process. Also, this paper addresses the tag prediction issue by recommending a personalized tag prediction probabilistic model. Personalized tag recommenders which take a user's previous tagging behavior into account when building recommendation usually have superior performance compared with general tag recommenders. The objective of CADS is to lower the cost of annotated document creation that can be instantly useful for commonly distributed semi-structured queries such as the ones and a personalized tag recommender is to tag prediction for each user specifically and effectively.

And also clustering technique is applied to integrate the subjects of items into the item-based collaborative filtering framework. The group rating evidence that is from the clustering consequence offers a way to present content information into collaborative recommendation and resolves the cold start difficulty (where recommendations are appropriate for items that no user has been rated).

The rest of the paper is formulated as follows: Section II represents a Literature review. Section III represents problem statement. In section IV addressed proposed System. And section V addressed computation strategy. And section VI represents Expected Result. Final Conclusion is defined in section VII.

\section{LITERATURE REVIEW \\ 2.1. Collaborative Annotation}

There are some techniques that provides the collaborative annotation of objects and use previous tags or annotations to annotate new objects. In paper [3], the authors predicted tags based on anchor and page content, close hosts, and other tags applied to the URL. After analysis they found an entropy-based metric which used to capture the summary of a specific tag and informs an examination of well tag prediction. Also constitutes that tag-based organization rules can harvest very high-precision anticipation also granting a deeper understanding into the relationships between tags.

Similarly, Y. Song et al. [4] proposed a highly-automated novel framework for real-time tag recommendation, including a Poisson mixture model for efficient document classification and node ranking method. But these techniques recommend tags in one second on average.

In paper [5], Douglas Eck et al. proposed an automatic social tag prediction system for music recommendation application using a set of boosting classifier. But this system is biased to favour popular artists.

In paper [6] authors described a web-based image annotation tool that was used to label the identity of objects and where they occur in images. They showed how to enhance and improve the aspect of the dataset through the application of WordNet, heuristics to repair item parts and depth ordering, and training of an item detector using the possessed labels to increase the size of data sets images repeated by online search engines.

\subsection{Dataspace Integration}

The integration model of CADS is like that of dataspaces [7], in which integration model is projected for heterogeneous sources. 
In paper [2] S.R. Jeffery et al. proposed a decision-theoretic methodology to require comment of users in a dataspace. They assested a service function that captures the service of a given dataspace state in terms of query result aspect.

Similarly, in paper [8], J. Madhavan et al. Propose novel data incorporation architecture called as PAYGO, which is encouraged by the concept of dataspaces and emphasizes pay-as-you-go records management [9] as a means for attaining web-scale data integration. Though, no previous work studies this issue at insertion period, as in CADS.

\subsection{Information Extraction (IE)}

Information extraction is relevant to creation which are required in the situation of a value suggestion for the computed attributes.

In paper [10] M.J. Cafarella et al. Describes three information extraction systems that can be operated on the entire Web.

Similarly, O. Etzioni et al. [11] presented an article for information retrieval system intended for finding instances of a specific relationship in the text using an open-ended technique (Open IE) which balances the entire Web and can also support a wide range of unanticipated questions over arbitrary associations. Open IE produces RDF-like triplets without any input from the user.

The Community Information Management CIMPLE project [12], [13] uses IE methods to make and manage data-rich online groups, called the prototype system like the DBLife community. In contradiction of Community Information Management Project CIMPLE, where data are removed from previous sources and an area expert must create a domain system, CADS is a document sharing environment where users explicitly insert the documents and the system automatically grows with time. However, the IE [18] and figure collaboration methods of CIMPLE can help in developing adaptive insertion forms in CADS.

\subsection{Query Forms}

In paper [14] M. Jayapandian and H. Jagadish recommends a system of citation a query form that characterizes maximum number of the queries in the database with the use of the "querability" of the columns. Whereas in [15] they extend their effort deliberating forms customization by evolving a query producer that modifies the form's novel query based on a user's variation.

A. Nandi and H. V. Jagdish [17] demonstrates a different query interface that allows users to build a rich search query with no any prior knowledge of the fundamental system or data. In this they use the structure information to autocomplete characters or content names in query forms.

In [13] E. Chu, used keyword queries to select the most suitable query forms. They also address challenges that rise in keyword search over forms, form generation, and data ranking and showing these forms.

\subsection{Probabilistic Model}

In paper [16], D. Liu et al. propose a tag ranking system that directing to automatically rank the tags related to a specified image according to their significance to the image contented.

Authors D. Yin et al. [17] address the difficulty of tag prediction by recommending a probabilistic model for personalized tag prediction. 


\section{Problem statement}

Document annotation technique facilitates the generation of the structured metadata that contain information of user's interest. But this existing system based on keyword base searching, which will lead to increase the number of clicks of users which may get irrelevant information or noisy data. This recommendation of any information may reduce effort of users to search using a keyword query which is not handled by existing system.

The existing system consists adaptive methods to suggest related attributes to annotate a document, while trying to fulfil the user querying requirements. The Existing system is used to search keywords that are entered by the user. But this does not contain recommendation predicted before search so that users may not need to search information.

\section{Proposed SYSTEM}

According to instance explained in [1], CADS technique is explained. The main objective of CADS is to produce and decline the cost of generating well annotated documents that can be directly useful for commonly issued semi-structured queries. The contribution of this paper is stated as follows:

- This paper presents an adaptive technique for automatically creating evidence input forms, aimed at annotating unstructured textual documents, like the use of the inserted data is exploited, given the user information requirements.

- This creates principled probabilistic methods and algorithms to easily integrate data from the query workload databases into the data or content annotation operations. This method creates metadata that are not applicable to the annotated document and not valuable to the users querying the database.

- This present extensive investigation with real data and real users, viewing that the system gives accurate results that are well superior to the suggestions for different approaches.

- This present a novel algorithm to map extracted data to standard format fields in the event schemas [16].

- A system is proposed, that presents the collaborative filtering to recover its prediction quality and resolve the difficulty of cold start [18].

Since the objective of the annotations is to facilitate future querying to focus on generating annotations which are useful for the queries in the query workload $\mathrm{W}$ that retrieve document $\mathrm{d}$.

- The attributes must have a high querying value (QV) regarding the query workload W. Viz., they must seem in numerous queries in $\mathrm{W}$, because the frequent attributes in $\mathrm{W}$ have a greater potential to improve the visibility of $d$.

- The attributes must have a high content value (CV) regarding data. Viz., they must be relevant to the data. Else, the user will perhaps dismiss the suggestions and $\mathrm{d}$ will not be correctly annotated.

- The attribute essentially has a frequent value (FV) rendering to query workload W [16]. Viz., they must have a highest mapping score with various factors, for example, type of document, location (in case of natural disaster) etc. Based on this match score, one can find the best schema attribute for each extracted attribute. 


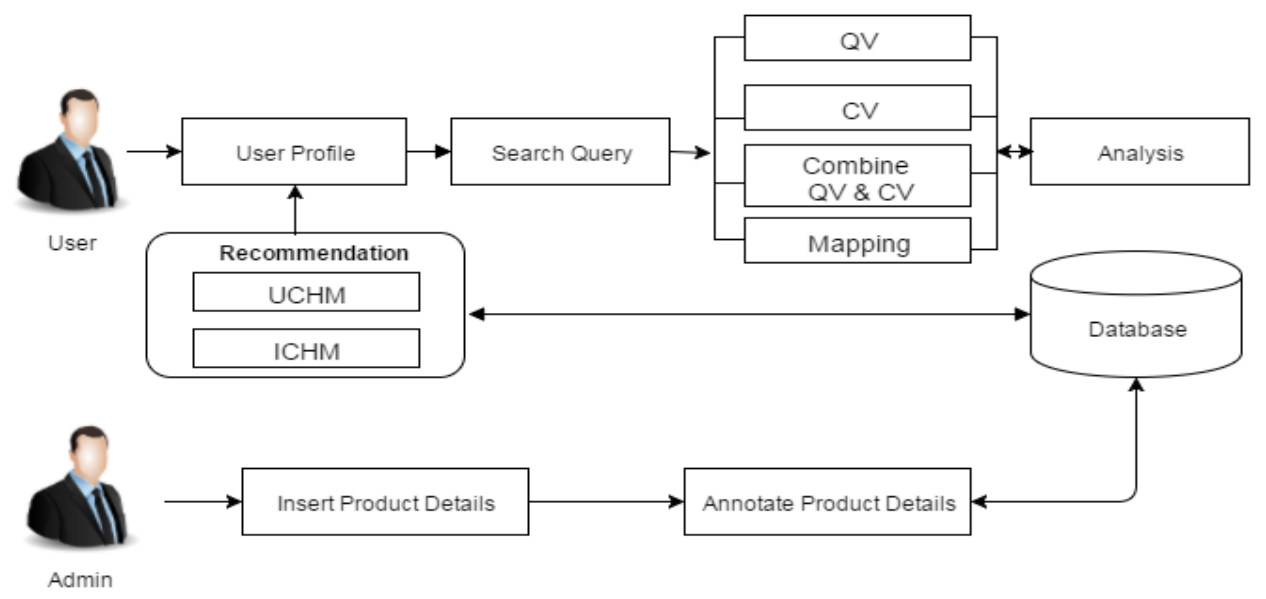

Figure 1. Proposed Architecture

\subsection{Proposed Architecture}

In the Existing System user can search keyword that is entered by the user, but this does not contain a recommendation predict before the search. To eliminate this drawback in the proposed system. Figure 1. Shows the proposed architecture, In this architecture Admin can insert the product detail into the database but before inserting the product detail into the database, annotate particular attributes or product detail.

When user login into the system before starting the search query it gets the recommended data from the database using UCHM and ICHM techniques. After that user can search the data using $\mathrm{QV}, \mathrm{CV}$, a combination of these two and Mapping Attribute value algorithm.

\section{COMPUTATION STRATEGIES}

This section discusses the mathematical approaches that allow to implement efficient algorithms.

\subsection{QV Computation}

A key analysis is that $Q V$ of an attribute is not depend on the acknowledged document; $Q V$ only depends on the query workload $W$. Therefore, a pre-computed list List $(Q V)$ of $Q V \mathrm{~s}$ of the attributes in the database with Attribute called as $D_{n}$, ordered by decreasing $Q V$ values is maintained. Subsequently the query workload does not change meaningfully in real time, thus update List $(Q D)$ only periodically, as next queries arrive, as it is not critical for the $q V$ metrics to be unconditionally up-to-date: approximations suffice.

Let $W_{A_{j}}=\left\{Q \in W_{\text {i use }}\left(Q, A_{j}\right)\right]$ Be the set of queries in $W$ that use $A_{j}$ Annotation as one of the predicate conditions [1]. Therefore the probabilities for the attributes which do not look in the workload are given as:

$$
p\left(A_{j} \mid W\right)=\frac{\left|W_{A_{j}}\right|+1}{|W|+1}
$$




\subsection{Computation}

Opposite to this, it is costly in terms of time and space to preserve all the Content Values for all pairs of the data, documents and content of the attribute, where, $C V$ (content value). To handle that, compute the $C V$ s at runtime whenever a document arrives.

For the content value $p\left(d_{t} \mid A_{j}\right)$, The probabilistic model assumes independence between the terms in $\mathrm{dt}$, which is a typical assumption when dealing with textual data, given as:

$$
p\left(d_{1} \mid A_{j}\right)=\prod_{w \in E_{1}} p\left(w \mid A_{j}\right)
$$

Where, the product goes over all terms $\mathrm{w}$ in dt. This is explained with example in paper [1].

\subsection{Combining of QV and CV Computation}

The algorithm executes as follows:

Step 1: Select Precomputed list of QV L(QV).

Step 2: Retrieve attribute $A$ : from $\mathrm{L}(\mathrm{QV})$.

Step 3: Compute threshold value $\mathrm{T}=F\left(\overline{C V}, Q V\left(A_{j}\right)\right)$

Where, $\overline{C V}$ is the content value of documents that are not annotated value or unseen attribute. $Q V\left(A_{j}\right)$ is the attribute Query value.

Step 4: Take $\mathrm{R}$ be the set of $\mathrm{k}$ attribute with highest score from $\mathrm{QV}$ and $\mathrm{CV}$ list of documents. Step 5: If $K^{\text {th }}$ attribute $A_{k}$ has score $\left(A_{k}\right)>\mathrm{T}$ then return R. Else go to Step 1.

$$
\text { Score }\left(A_{k}\right)=\frac{P\left(A_{k} \mid W\right)}{1-P\left(A_{k} \mid W\right)} \cdot \frac{P\left(\text { dr } \mid A_{k}\right)}{P\left(d t \mid A_{R}\right)}
$$

Where, $\quad \mathrm{P}\left(d t \mid A_{k}\right)=\prod_{w} \subset d t P\left(w \mid A_{k}\right)$

\subsection{Mapping Attribute-Value Computation}

This algorithm used to compute mapping score between the attribute and value pair present in every schema. In this paper, the match score depends on the similarity between the frequent values of attributes. The steps for mapping algorithm are:

Assume that structured data having attributes: type, group rating in percentage, the number of people visited for the product, location for product availability.

Step1: Let, query consist value $(q)$ for any $A_{j}$ annotation of documents $d$, mapping score $=0$.

Step2: For every product $p$ in $W$.

Step3: If $q$ contains product type then

$$
\text { Score }=\text { Score }+1
$$

Step4: If $q$ contains price $>\min$ and price $>\max \quad$ (min, max refers to product price)

$$
\text { Score }=\text { Score }+1
$$

Step5: If $q$ contains product rating ( $p r)$ then

$p r \geq T$ ( $T$ is average rating of product $P$ )

$$
\text { Score }=\text { Score }+1
$$


Step6: If $q$ contains location then

If query location $(q l) \in$ Product location $(p l)$

$$
\begin{gathered}
\operatorname{sim}(q l, p l)=1 \\
\text { Score }=\text { Score }+2 *(\operatorname{sim}(q l, p l))
\end{gathered}
$$

Else

If query location $(q l) \notin$ Product location $(p l)$

$$
\begin{aligned}
& \operatorname{sim}(q l, p l)=0 \\
& \text { Score }=\text { Score }+2 *(\operatorname{sim}(q l, p l))
\end{aligned}
$$

Step7: Find the result with max score $S$

In above algorithm, the type of event is checked with the query, for instance, the product information extraction. If that type of particular product (e.g. Electronics product, home appliances, etc.) equalizes to query value, then the score is increased by 1 . Similarly, in step4 min and max are used for minimum and a maximum price of a product respectively. In step5, T is the threshold that, it is computed by average product rating for the particular product given by the group of users. Step 6 gives query content associated with the location of a dealer (dealer id a product provider). If there is the similarity between locations then the similarity is 1 else 0 . Finally, the document which gives maximum score will be predicted to user as search query result.

\subsection{Product Recommendation}

Clustering technique is applied to the item content information to complement the user rating information, which improves the correctness of collaborative similarity, and solves the cold start problem. For collaborative clustering, the Pearson-correlation based similarity and adjusts the cosine similarity is used.

\subsubsection{Pearson Correlation-based Similarity}

$$
\operatorname{sim}\left(k_{i} l\right)=\frac{\operatorname{cov}\left(k_{,} l\right)}{\sigma_{k} \sigma_{l}}=\frac{\sum_{u=l}^{m}\left(R_{u, k}-\bar{R}_{k}\right)\left(R_{u, l}-\bar{R}_{l}\right)}{\sqrt{\sum_{u=l}^{n}\left(R_{u, k}-\bar{R}_{k}\right)^{2}} \sqrt{\sum_{i=l}^{n}\left(\bar{R}_{u, !}-\bar{R}_{l}\right)^{2}}}
$$

Where,

$\operatorname{sim}(k, l)$ means the similarity between item $k$ and $l, n$ means the total number of users, who graded on both item $k$ and $l, \bar{R}_{k}, \bar{R}_{l}$ are the average ratings of the item $k$ and $l$, respectively; $\mathbb{R}_{u, k i}, R_{u, l}$ mean the rating of user $u$ on items $k$ and $l$ respectively.

\subsubsection{Adjusted Cosine Similarity}

$$
\operatorname{sim}(k, D)=\frac{\sum_{u=1}^{n}\left(R_{u, k}-\bar{R}_{u}\right)\left(R_{u l}-\bar{R}_{u}\right)}{\sqrt{\sum_{u=l}^{n}\left(\bar{R}_{u, k}-\bar{\kappa}_{u}\right)^{2}} \sqrt{\sum_{i=1}^{n}\left(\bar{R}_{u l}-\bar{\kappa}_{u}\right)^{2}}}
$$

Where,

$\operatorname{sim}(k, b)$ means the similarity between item $\mathrm{k}$ and $1 ; n$ means the total number of users, who graded on both item $\mathrm{k}$ and $\mathrm{l} ; \overline{\boldsymbol{R}}_{u}$ are the average ratings of user $\mathrm{u} ; \boldsymbol{R}_{u_{i}, \bar{l}}, \boldsymbol{R}_{u_{l} l}$ mean the rating of user $u$ on items $k$ and $l$ respectively. 


\subsubsection{Linear Combination}

$$
\operatorname{sim}(k, D)=\sin \left(k, b_{\text {tem }} \times(1-c)+\operatorname{sim}\left(k, b_{\text {growg }} \times c\right.\right.
$$

Where,

$c$ Means the combination coefficient, $\operatorname{sim}\left(k, b_{\tan }\right.$ Means that the similarity between item $k$ and $l$, $\operatorname{sim}(k, l)_{\text {growg }}$ Means that the similarity between item $k$ and $l$.

\subsubsection{Collaborative Prediction}

Prediction for an item is then computed by

Where,

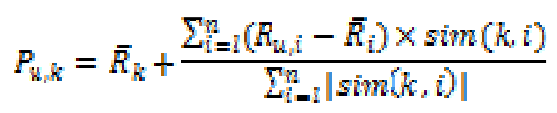

$\boldsymbol{P}_{u, k}$ represents the predication for the user u on item k; n means the total neighbours of item $\mathrm{k} ; \boldsymbol{R}_{u, i}$ means the user u rating on item $\mathrm{i} ; \bar{R}_{k}$ is the average ratings on item k ; $\operatorname{sim}\left(k_{s} i\right)$ means the similarity between item $\mathrm{k}$ and its' neighbour i; $\boldsymbol{R}_{i}$ means the average ratings on item $i$.

\section{EXPECTED RESULT}

\subsection{Recommendation Evaluation}

MAE that is Mean Absolute Error [19] has usually used in calculating the accuracy of a recommender system by comparison the numerical recommendation scores against the particular user ratings within the test data. The MAE is calculated by summing these absolute errors of the respective rating prediction pairs and then computing the average.

$$
M A E=\frac{\sum_{u-1}^{n}\left|P_{u, i}-R_{u, i}\right|}{n}
$$

Where,

$P_{u, i}$ Means the user u prediction on the item $i ; R_{u, i}$ means the user $u$ rating for an item $i$ in the test data; $n$ is the number of rating prediction pairs between the test data and the prediction result. The result will more accurate if the value of MAE is lower.

The value of MAE will be computed using the prediction $\mathrm{P}$ and actual $\mathrm{R}$ rating. For any user, if set of products is recommended then that recommendation is depends on these predicted and real ratings given to products. MAE is calculated for both item-based and user-based methods.

\subsection{Attribute Suggestion Evaluation}

QV suggest attributes based on the querying value component of workload W. CV suggests attributes based on the content value component. Attribute suggestion results for the combination of $\mathrm{QV}, \mathrm{CV}$ and mapping algorithm are expected as shown in the table given below. 
International Journal of Managing Public Sector Information and Communication Technologies (IJMPICT) Vol. 7, No. 2, June 2016

Table 1. Expected Result of Algorithm

\begin{tabular}{|c|c|c|c|c|c|c|}
\hline \multirow[t]{2}{*}{ W } & \multirow{2}{*}{$\begin{array}{c}\text { No. of } \\
\text { doc }\end{array}$} & \multicolumn{4}{|c|}{ Predicted Annotate Documents } & \multirow{2}{*}{$\begin{array}{c}\text { Actual } \\
\text { Annotate } \\
\text { Documents }\end{array}$} \\
\hline & & OV & $\mathrm{CV}$ & $\mathrm{OV} \& \mathrm{CV}$ & Map & \\
\hline W1 & 50 & 16 & 16 & 17 & 18 & 20 \\
\hline $\mathrm{W} 2$ & 100 & 39 & 40 & 52 & 40 & 57 \\
\hline W3 & 150 & 98 & 91 & 90 & 99 & 99 \\
\hline W4 & 200 & 58 & 50 & 78 & 85 & 90 \\
\hline W5 & 250 & 50 & 54 & 58 & 59 & 68 \\
\hline
\end{tabular}

As shown in Table 1. The retrieval results by applying all four methods are given. For example, considering first scenario, Let database is having 50 numbers of documents. And W1 is workload for the query. For any query in workload if there are 20 actual entries in the database related to keywords, then, according to algorithm suppose out of 20 documents, 16 documents are retrieved by applying QV, 16 retrieved by $\mathrm{CV}, 17$ retrieved by a combination of $\mathrm{CV}$ and $\mathrm{QV}$, and 18 retrieved from mapping algorithm. Similarly, the results are computed for 100, 150, 200, and 250 document entries.

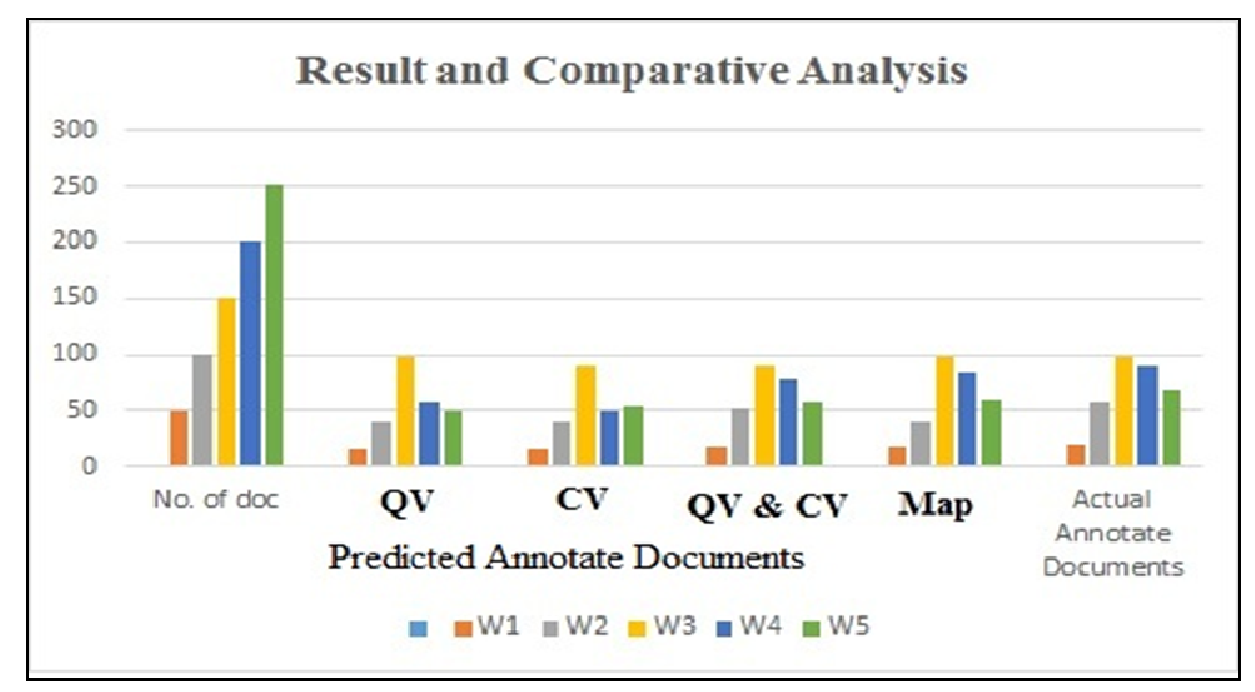

Figure 2. Comparative Analysis of various Techniques for Annotation

\section{CONCLUSIONS}

This paper proposed mapping attributes value algorithm that deliberates the suggestion in the content of the document and the query workload database. It extracts data with computation of score of frequent value of attribute. Thus we implement document annotation using content value, querying value, a combination of these two and mapping attribute value to annotate a document. This paper also focuses on collaborative filtering approach in some manner to recommend any user data previously. 
International Journal of Managing Public Sector Information and Communication Technologies (IJMPICT) Vol. 7, No. 2, June 2016

\section{ACKNOWLEDGEMENTS}

We would like to thank the researchers as well as publishers for making their resources available and teachers for their guidance. The authors would like to thank the Department of Computer Engineering of D. Y. Patil School of Engineering and Technology, Pune, India for their generous support. They would also like to thank the Principal Dr. Ashok Kasnale, HOD Ms. Arti Mohanpurkar and all Staff Members of Computer Engineering department for their valuable guidance.We are also thankful to reviewer for their valuable suggestions and also thank the college authorities for providing the required infrastructure and support.

\section{REFERENCES}

[1] A.Y. Halevy, S.R. Jeffery, and M.J. Franklin, "Pay-as-You-Go User Feedback for Dataspace Systems," Proc. ACM SIGMOD Int'l Conf. Management Data, 2008.

[2] H. Garcia-Molina ,P. Heymann, and D. Ramage, "Social Tag Prediction,” Proc. 31st Ann. Int'l ACM SIGIR Conf. Research and Development in Information Retrieval (SIGIR '08), pp. 531-538, 2008.

[3] Y. Song, Z. Zhuang, W. C. Lee, H. Li, C.L. Giles and J. Li Q. Zhao, "Real-Time Automatic Tag Recommendation," Proc. 31st Ann. Int'l ACM SIGIR Conf. Research and Development in Information Retrieval (SIGIR '08), pp. 515-522, 2008.

[4] D. Eck, T. Bertin-Mahieux, P. Lamere, and S. Green, "Automatic Generation of Social Tags for Music Recommendation,” Proc. Advances in Neural Information Processing Systems 20, 2008.

[5] B. Russell, K. Murphy, A. Torralba, and W. F., "Label Me: A Database and Web-Based Tool for Image Annotation,” Int'1 J. Computer Vision, vol. 77, pp. 157-173, 2008.

[6] M. Franklin, D. Maier, A. Halevy., "From Databases to Dataspaces: A New Abstraction for Information Management," SIGMOD Record, vol. 34, pp. 27-33, Dec. 2005.

[7] . Madhavan, Shawn R. Jeffery, Shirley Cohen, Xin (Luna) Dong, David Ko, Cong Yu, Alon Halevy, "Web-Scale Data Integration: You Can Only Afford to Pay as You Go," Proc. Third Biennial Conf. Innovative Data Systems Research (CIDR), 2007.

[8] A. Halevy, D. Suciu, Z. Ives, and I. Tatarinov, "Schema Mediation in Peer Data Management Systems," Proc. 19th Int'1 Conf. Data Eng., pp. 505-516, Mar. 2003.

[9] J. Madhavan, M.J. Cafarella, and A. Halevy, "Web-Scale Extraction of Structured Data, SIGMOD Record," vol. 37, pp. 55-61, Mar. 2009.

[10] O. Etzioni, D.S. Weld, M. Banko, and S. Soderland, "Open Information Extraction from the Web,"Comm. ACM, vol. 51, pp. 68-74, Dec. 2008.

[11] R. Ramakrishnan, F. Chen, P. DeRose, A. Doan, R. McCann, M. Sayyadian, Y. Lee, and W. Shen, "Community Information Management," IEEE Data Eng. Bull., vol. 29, no. 1, pp. 64-72, Mar. 2006.

[12] E. Chu, A. Baid, A. Doan, X. Chai, and J. Naughton, "Combining Keyword Search and Forms for Ad Hoc Querying of Databases,” Proc. ACM SIGMOD Int'l Conf. Management Data, 2009.

[13] H.V. Jagadish, and M. Jayapandian, "Automated Creation of a Forms-Based Database Query Interface,” Proc. VLDB Endowment, vol. 1, pp. 695-709, Aug. 2008.

[14] H. Jagadish, and M. Jayapandian, "Expressive Query Specification through Form Customization," Proc. 11th Int'l Conf. Extending Database Technology: Advances in Database Technology (EDBT '08), pp. 416-427, 2008.

[15] S. Panem, V. Varma, and M. Gupta, "Structured Information Extraction from Natural Disaster Events on Twitter," 23nd International Conf. On Information and Knowledge Management November 2014.

[16] H.V. Jagadish, and A. Nandi, “Assisted Querying Using Instant Response Interfaces,” Proc. ACM SIGMOD International Conf. Management Data, 2007

[17] Qing Li, and Byeong Man Kim, "An Approach for Combining Content-based and Collaborative Filters," Korea Research Foundation Grant, 2003.

[18] Priyanka A. Channe and Bhagyashree Dhakulkar, "A Review on Document Annotation Technique," International Journal of Computer Applications (IJCA) Proceedings on National Conference on Advances in Computing NCAC-2015(4): 19-22, December 2015 (ISSN : 0975-8887).

[19] Priyanka A. Channe and Bhagyashree Dhakulkar, "Document Annotation for Effective Structured Data Information Retrieval," Ciit International Journal of Artificial Intelligent System and Machine Learning, Volume 8, No. 3, March 2016 ( ISSN: 0974 - 9543). 
International Journal of Managing Public Sector Information and Communication Technologies (IJMPICT) Vol. 7, No. 2, June 2016

\section{Authors}

Ms. Priyanka Channe received Diploma in Computer Engineering in year 2010 and Bachelor of Engineering degree in Computer Engineering in 2013 and now pursuing Post Graduation (M.E.) in the department of Computer Engineering from Dr. D. Y. Patil School of Engineering and Technology in the current academic year 2015-16. She is now studying for the domain Data Mining and Information Retrieval as research purpose on Document Annotation during his academic year.

Prof. Bhagyashree Dhakulkar. She is presently working as an Assistant. Professor, Department of Computer Engineering, Dr. D. Y. Patil School Of Engineering and Technology, Charoli, B. K. Via -Lohegaon, Pune, Maharashtra, India. She has 11 year experience in teaching field and her research area is Data Mining and Information Retrieval.
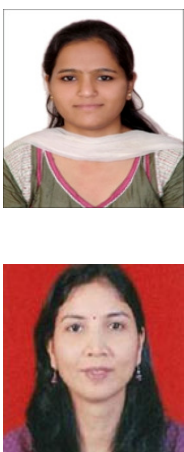